\title{
First-principles Calculation of the Single Impurity Surface Kondo Resonance
}

\author{
Chiung-Yuan Lin, ${ }^{1,2}$ A. H. Castro Neto, ${ }^{1}$ and B. A. Jones ${ }^{2}$ \\ ${ }^{1}$ Department of Physics, Boston University, 590 Commonwealth Avenue, Boston, Massachusetts 02215, USA \\ ${ }^{2}$ IBM Almaden Research Center, San Jose, CA 95120-6099, USA
}

(Dated: 28th September 2018)

\begin{abstract}
We have performed first-principles calculation of the surface and bulk wavefunctions of the $\mathrm{Cu}(111)$ surface and their hybridization energies to a Co adatom, including the potential scattering from the Co. By analyzing the calculated hybridization energies, we found the bulk states dominate the contribution to the Kondo temperature, in agreement with recent experiments. Furthermore, we also calculate the tunneling conductance of a scanning tunneling microscope (STM) and compare our results with recent experiments of $\mathrm{Co}$ impurities in the $\mathrm{Cu}(111)$ surface. Good quantitative agreement is found at short parallel impurity-tip distances $(<6 \AA)$. Our results indicate the need for a new formulation of the problem at larger distances.
\end{abstract}

PACS numbers: 68.37.Ef, 72.15.Qm, 72.10.Fk

Scanning tunneling microscopy (STM) has become one of the most basic tools for the manipulation of matter at the atomic scale. Although this experimental technique has reached maturity, the detailed theoretical understanding of experimental data is still incomplete and/or contradictory. One of the most famous examples of atomic manipulation is associated with the surface Kondo effect observed when transition metal ions (such as $\mathrm{Co}$ ) are placed on a metallic surface (such as $\mathrm{Cu}(111)$ ) 1]. The surface Kondo effect is the basis for the observation of surprising phenomena such as quantum mirages 2], and has attracted a lot of attention and interest in the last few years. The current understanding of these observations is based on the assumption that only surface states of $\mathrm{Cu}(111)$ are involved in the scattering of electron waves by the Co adatoms [3, [4, [5]. Nevertheless, recent experiments with $\mathrm{Co}$ atoms on the $\mathrm{Cu}(100)$ surface (that does not have any surface state) [6], or in $\mathrm{Cu}(111)$ but close to atomic surface steps (that affect the surface states) 7] have indicated that bulk (not surface) states are behind the surface Kondo effect. Meanwhile, in contrast, the growing theoretical literature in the subject is heavily concentrated on the surface states alone. In this paper, we use first-principles methods that clearly show that the bulk states are behind the surface Kondo effect, in agreement with these experiments. In the light of these results, theoretical approaches based on surface states alone have to be reconsidered.

The surface Kondo effect without the STM probe is described by the Anderson impurity model:

$$
\begin{aligned}
H_{s}= & \sum_{\mathbf{k} \sigma} \epsilon_{\mathbf{k}} c_{\mathbf{k} \sigma}^{\dagger} c_{\mathbf{k} \sigma}+\sum_{\mathbf{k} \sigma}\left(t_{\mathbf{k} a} c_{\mathbf{k} \sigma}^{\dagger} c_{a \sigma}+\text { H.c. }\right) \\
& +\sum_{\sigma} \epsilon_{a} c_{a \sigma}^{\dagger} c_{a \sigma}+U n_{a \uparrow} n_{a \downarrow}
\end{aligned}
$$

where $\epsilon_{\mathbf{k}}$ is the energy of the substrate electrons, $c_{\mathbf{k} \sigma}^{\dagger}$ $\left(c_{\mathbf{k} \sigma}\right)$ creation (annihilation) for substrate electrons with momentum $\mathbf{k}$ and spin $\sigma, \epsilon_{a}$ is the energy of the adatom $d$-orbital, $c_{a \sigma}^{\dagger}\left(c_{a \sigma}\right)$ is the creation (annihilation) of adatom electrons, and $U$ is the Coulomb energy for double occupancy of the $d$-orbital ( $n_{a \sigma}$ is the number operator). The hybridization energy between the substrate and adatom is written in terms of matrix elements between their wavefunctions.

$$
t_{\mathbf{k} a}=\int d^{3} r \psi_{\mathbf{k}}^{*}(\mathbf{r})\left(H_{0}+V_{a}(\mathbf{r})\right) \psi_{a}(\mathbf{r}),
$$

where $H_{0}$ refers to the bare metal surface, $V_{a}(r)$ is the adatom scattering potential, $\psi_{\mathbf{k}}(\mathbf{r})$ is the substrate wavefunction scattered by the adatom potential, and $\psi_{a}(\mathbf{r})$ the adatom $3 d$ orbital. Most work on the surface Kondo effect [8, 9] follows Anderson's original idea [10] in dealing with the hybridization matrix element $t_{\mathbf{k} a}$, namely, treating it as a phenomenological parameter to fit experiments. However, in trying to understand the STM experiments, and especially the role played by the surface and bulk states, these matrix elements cannot be simply taken as phenomenological parameters since one would not be able to separate the contributions coming from the bulk and the surface states of the substrate. Thus, it is essential to perform microscopic calculations of these matrix elements starting from the electronic wavefunctions.

Microscopic calculations of these matrix elements using the nearly free-electron model (NFE) for $\mathrm{Cu}$ have been attempted recently [1, 12]. The NFE approximation has its advantages in analyzing the momentum dependence of the hybridization energies and obtaining analytical substrate wavefunctions. However, in using the NFE one needs to prescribe how the substrate crystal potential joins its vacuum image counterpart, and the uniqueness of this potential prescription is questionable. Such a freedom in modeling potential makes the NFE method unreliable in obtaining the precise surface and bulk wavefunctions. Moreover, we further notice that the substrate states in (11) are those already scattered by the adatom potential rather than the bare-substrate states. The relation between these adatom-scattered states and the bare 
substrate states is given by:

$$
\begin{aligned}
\sum_{\mathbf{k} \sigma} \epsilon_{\mathbf{k}} c_{\mathbf{k} \sigma}^{\dagger} c_{\mathbf{k} \sigma} & =\sum_{\mathbf{k} \sigma} \epsilon_{\mathbf{k}} c_{\mathbf{k} \sigma}^{(0) \dagger} c_{\mathbf{k} \sigma}^{(0)} \\
& +\sum_{\mathbf{k} \mathbf{k}^{\prime} \sigma}\left(U_{\mathbf{k k}^{\prime}} c_{\mathbf{k} \sigma}^{(0) \dagger} c_{\mathbf{k}^{\prime} \sigma}^{(0)}+H . c .\right) \\
U_{\mathbf{k k}^{\prime}} & =\int d^{3} r \psi_{\mathbf{k}}^{(0) *}(\mathbf{r}) V_{a}(\mathbf{r}) \psi_{\mathbf{k}^{\prime}}^{(0)}(\mathbf{r}),
\end{aligned}
$$

where the superscript (0) refers to the bare electronic states. The NFE does not consider the scattering potential from the adatom and uses the bare-substrate states in the Anderson impurity model (1).

In the presence of the tip new terms have to be added to the Hamiltonian that describe the tunneling processes of tip-to-adatom and tip-to-substrate [9]. The tip-to-adatom tunneling process is described by: $H_{a t}=$ $\sum_{\sigma} t_{a p}\left(c_{a \sigma}^{\dagger} c_{p \sigma}+\right.$ H.c.), where $c_{p \sigma}\left(c_{p \sigma}^{\dagger}\right)$ annihilates (creates) electrons at the tip and $t_{a p}$ is the hybridization energy between tip and adatom. The tip-to-substrate hybridization is given by: $H_{s t}=\sum_{k \sigma} t_{\mathbf{k} p}\left(c_{\mathbf{k} \sigma}^{\dagger} c_{p \sigma}+\right.$ H.c. $)$, where $t_{\mathrm{k} p}$ is the hybridization energy between substrate (bulk or surface state) and adatom. The tip Hamiltonian is simply: $H_{t}=\sum_{\sigma} \epsilon_{p} c_{p \sigma}^{\dagger} c_{p \sigma}$, where $\epsilon_{p}$ is the energy of the tip electrons. The total Hamiltonian of the tipsubstrate-adsorbate system is: $H=H_{s}+H_{t}+H_{a t}+H_{s t}$. For the hybridization energies that involve the STM tip, we follow Plihal and Gadzuk [9] and approximate $t_{p \alpha} \propto \psi_{\alpha}^{*}\left(\mathbf{R}_{t}\right)$ with $\alpha=\mathbf{k}, a$.

To correctly obtain the contributions of the surface Kondo resonance from the surface and bulk states, we perform first-principles calculations of surface and bulk wavefunctions on the $\mathrm{Cu}(111)$ surface in the presence of the scattering potential, as well as their hybridization energies to the Co adatom. As the first step, we calculate the wavefunctions of a bare $\mathrm{Cu}(111)$ surface. Such a surface is simulated by a super-cell of 21-layer slabs separated by 8 vacuum layers. We employ, in the framework of density functional theory, a self-consistent fullpotential linearized augmented plane wave (FLAPW) method [13], with the exchange-correlation potential in the generalized gradient approximation (GGA) 14]. The interatomic distances within a $\mathrm{Cu}$ slab are determined by the bulk lattice constant $a_{0}=3.62 \AA$, and the surface relaxations of $\mathrm{Cu}(111)$ are neglected because of its closepacked structure. The calculated surface-state dispersion agrees very well with the experiments (Fig. 1 left).

In order to obtain the potential of a Co adatom, we perform another FLAPW calculation, in the local spin density approximation (LSDA), of 7-layer Cu slabs separated by 8 vacuum layers, plus Co impurities 1-layer spacing high on top of the $\mathrm{Cu}$ surface layers (Fig. 1 right). The potential $V_{a}(\mathbf{r})$ appearing in both (2) and (4) is taken to be the potential difference between the surface with $\mathrm{Co}$ and the clean crystal. Using the bare $\mathrm{Cu}(111)$ states within the energy range $\left|\epsilon-\epsilon_{F}\right|<1 \mathrm{eV}$ as basis, we per-

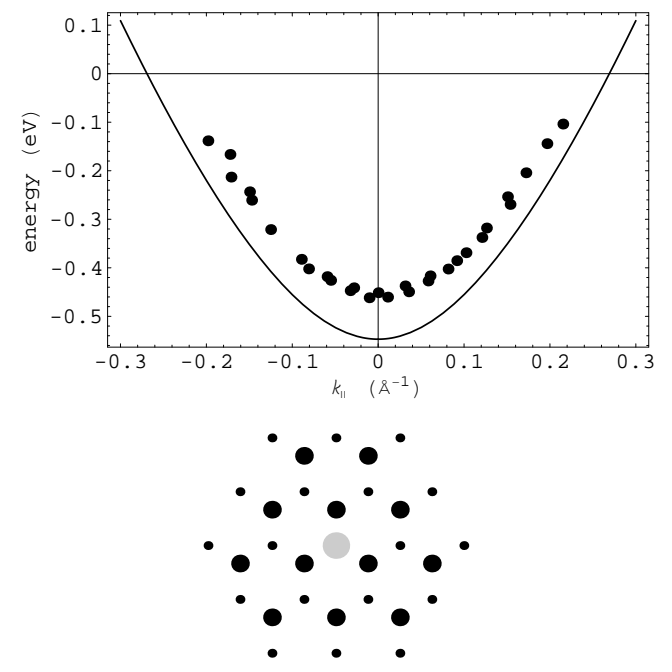

Figure 1: The left figure shows the surface-state dispersion from our calculation (curve) and the experiments [15] (data points). The right figure shows the top view of the unit cell of $\mathrm{Co}$ on the $\mathrm{Cu}(111)$ surface in our LSDA calculation of the present work. The Co atom is located at the center (grey circle). The larger and smaller circles are the first (surface) and second $\mathrm{Cu}$ layers, respectively. The individual Co atoms are separated by $8 \AA$.

form an exact matrix diagonalization on the right-hand side of (3) and obtain the adatom scattered states. It is the $3 d$ orbital of the Co adatom that actually participates in the Kondo resonance. The $3 d$ orbital $c_{a \sigma}$ appearing in (11) is renormalized by the $\mathrm{Cu}$-substrate potential but does not include hybridization with the substrate states. Our current first-principles approach cannot generate $3 d$ orbitals satisfying both conditions. Instead we can perform LSDA calculations for the $3 d$ orbital of either a single $\mathrm{Co}$ atom or a $\mathrm{Co}$ atom on $\mathrm{Cu}(111)$ with hybridization $t_{a \mathbf{k}}$ included. The latter is not a good candidate to be used in (11) because it adsorbs $t_{a \mathbf{k}}$ into itself and should in principle give $t_{a \mathbf{k}} \approx 0$. Thus we calculate the electronic structure of a single Co atom using a relativistic atomic code [16] and use its $3 d_{3 z^{2}-r^{2}}$ orbital as the $\psi_{a}(\mathbf{r})$ in (2). The particular choice of the $3 d$ orbital $3 d_{3 z^{2}-r^{2}}$ other than $3 d_{x y}$ or $3 d_{x^{2}-y^{2}}$ is supported by Ref. [17].

The theory of the surface Kondo resonance adopted in the present work closely follows Ref. 11, 18. The broadening of the Co $d$-level is calculated directly from $t_{\mathbf{k} a}$ in (2) (without any adjustable parameters)

$$
\Delta=\pi \sum_{\mathbf{k}}\left|t_{\mathbf{k} a}\right|^{2} \delta\left(\epsilon_{F}-\epsilon_{\mathbf{k}}\right),
$$

where $\epsilon_{F}$ is the Fermi energy, to be $\Delta=0.18 \mathrm{eV}$. In fact, the STM-measured Kondo temperature for $\mathrm{Co} / \mathrm{Cu}(111)$ is $T_{K} \sim 50 K$, which, from $T_{K}=D \exp \left(-\pi\left|\epsilon_{a}\right| / \Delta\right)$, gives $\Delta \sim 0.2 \mathrm{eV}$ if one uses well established values of the $d$ level $\epsilon_{a} \sim 0.9 \mathrm{eV}[\underline{8}]$ and the $\mathrm{Cu}$ band cutoff $D \sim$ 
$5.5 \mathrm{eV}[19]$. The contributions to $\Delta$ from the surface and bulk states are also investigated by considering $\Delta=$ $\Delta_{\text {surf }}+\Delta_{\text {bulk. }}$. We calculate from (5) the ratio of the surface-state contribution to the total $d$-level broadening $\Delta_{\text {surf }} / \Delta \sim 0.006$. The ratio shows that the bulk states dominate the adatom-substrate hybridization energy of $\mathrm{Co} / \mathrm{Cu}(111)$. This result can be understood by the fact that the Co atom is still in one layer above the surface $\mathrm{Cu}$ layer, a crystal-like regime rather than the tunneling regime (that is, the Co adatom is strongly hybridized with the substrate).

The STM differential conductance can be written as:

$$
\frac{d I}{d V}-\left(\frac{d I}{d V}\right)_{0}=a(R) \frac{|q(R)|^{2}-1+2 \xi \operatorname{Re}[q(R)]}{\xi^{2}+1} .
$$

where $R$ is the parallel impurity-tip distance, $\xi=(\mathrm{eV}+$ $\left.\bar{\epsilon}_{a}\right) /\left(k_{B} T_{K}\right)$ is the dimensionless bias, $\bar{\epsilon}_{a}$ is a bias off-set due to the $d$-state energy,

$$
a(R)=\left|\pi \sum_{\mathbf{k}} t_{p \mathbf{k}} t_{\mathbf{k} a} \delta\left(\epsilon_{F}-\epsilon_{\mathbf{k}}\right)\right|^{2},
$$

is the amplitude of the resonance, and

$$
q=\frac{t_{p a}+\sum_{\mathbf{k}} t_{p \mathbf{k}} t_{\mathbf{k} a} \operatorname{Re} G_{\mathbf{k}}}{\sum_{\mathbf{k}} t_{p \mathbf{k}} t_{\mathbf{k} a} \operatorname{Im} G_{\mathbf{k}}}
$$

is the so-called Fano parameter where $G_{\mathbf{k}}=\left(\epsilon_{F}-\epsilon_{\mathbf{k}}+\right.$ $i \eta)^{-1}$ is the bare substrate Green's function. In (6), the differential conductance with a subscript "0" refers to the background signal (proportional to the local density of states of the substrate). Typically $q(R)$ has been taken to be real in the experimental fits. However, when performing the first-principles calculation of the $\mathrm{Cu}(111)$ surface, we found that $q$ can carry an imaginary part. The Bloch states of the conduction electrons can generally be written as $\psi_{n \mathbf{k}}(\mathbf{r})=u_{n \mathbf{k}}(\mathbf{r}) e^{-i \mathbf{k}_{\|} \cdot \mathbf{r}_{\|}}$ with $u_{n \mathbf{k}}(\mathbf{r})=\left|u_{n \mathbf{k}}(\mathbf{r})\right| e^{i \Phi(\mathbf{r})}$. NFE studies of the surface Kondo resonance 9, 11, 12 treat $u_{n \mathbf{k}}$ in approximation such that $\Phi(\mathbf{r})$ is spatially independent, i.e., $\Phi(\mathbf{r})=\Phi_{0}$ is an overall phase. However, our first-principles calculation shows that the function $u_{n \mathbf{k}}$ can carry a spatially varying phase. Including $\Phi(\mathbf{r})$ in the calculation of the hybridization energies consequently gives the complex $q$.

The STM tip is positioned about $5 \AA<z<10 \AA$ above the surface in the usual spectroscopic tunneling conditions. However, when performing first-principles calculations of the bare- $\mathrm{Cu}(111)$ wavefunctions, we find that the wavefunctions undergo an oscillatory behavior rather than a smooth exponential decay beyond $4 \AA$ from the surface. It is known that this problem comes from GGA in the low density region because of large-scaled gradients 20]. The large gradients cause fluctuations in the exchange-correlation potential, which leads to fluctuations in wavefunctions. To resolve this problem we extrapolate the $\mathrm{Cu}$-substrate wavefunctions by fitting their

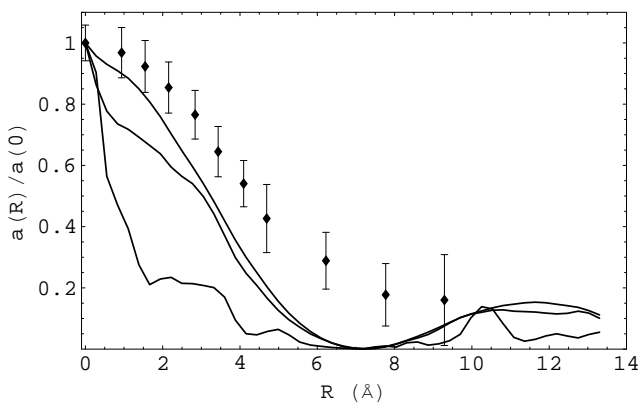

Figure 2: Normalized Fano formula prefactors $a(R) / a(0)$ at three tip heights $Z_{t}=3.5 \AA$, $10 \AA, 16 \AA$ (from the bottom to the top). The experimental data is also shown [6].

values in $2 \AA<z<3 \AA$ using the NFE wavefunctions [1]. The NFE wavefunction is then used in the $z>5 \AA$ region to calculate the STM differential conductance.

We analyze surface and bulk-state contributions to $a(R)$, and find that the bulk states dominate. In Fig. 2 we plot $a(R)$ of three tip heights $\left(Z_{t}=3.5 \AA, 10 \AA\right.$, $16 \AA$ ) as well as the experimental data [6]. The prefactor $a(R)$ at $Z_{t}=3.5 \AA$ is calculated directly from the GGA wavefunctions rather than the extrapolated NFE wavefunctions. It is clear that $a(R)$ moves towards the experimental data as the tip moves farther away from the surface. One can see that our first-principles calculation at $Z_{t}=16 \AA$ (without fitting parameters) agrees well with the experiment within $R<5 \AA$ but starts deviating from the experiments as $R$ increases and has a node around $R=7 \AA$. Since the current first-principles approach in calculating the $\mathrm{Cu}(111)$ electronic structure, GGA, is widely regarded as a highly accurate computation scheme except for very low electron density, we suggest that further theoretical and experimental work is required to check this issue. In the bulk Kondo problem the conduction-impurity hybridization can be regarded as momentum-independent, and the Kondo Hamiltonian has an exact solution by Bethe ansatz 21. However, the surface Kondo effect has a $\mathbf{k}$-dependent $t_{\mathbf{k} a}$, and there is so far no field-theoretical approach that can treat it exactly. A possible solution is to use a computational scheme to compute the Anderson Hamiltonian of a surface Kondo effect. In the experimental aspect it should be pointed out that at large values of $R$ the STM data is very noisy, and it is possible that the fitting is not unique.

The calculation of $a(R)$ requires only the $\mathrm{Cu}$-substrate states at the Fermi energy (see Eq. (7)) while the lineshape parameter $q$ in (8) depends on the entire $\mathrm{Cu}$ band. We use the calculated Co-scattered $\mathrm{Cu}$ states within the energy range $\left|\epsilon-\epsilon_{F}\right|<0.9 \mathrm{eV}$ to obtain the $q(R)$ in Eq. (8). Since the Fano lineshape parameter $q$ defined in (8) is in general complex, the experimentally fitted $q$ based on the Fano formula of (8) with $\operatorname{Im}[q]=0$ can only 


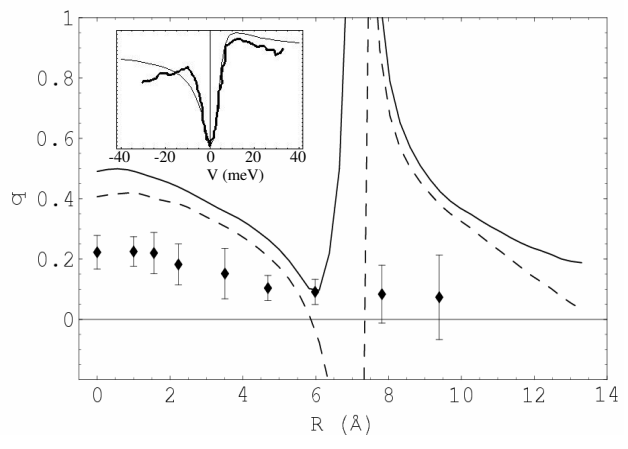

Figure 3: The absolute value (solid curve) and real part (dashed curve) of calculated complex $q$ at $Z_{t}=16 \AA$ as a function of $R$ as compared with the experimental fit assuming $\operatorname{Im}[q]=0$. Inset: comparison of theoretical (light) and experimental (dark) STM $d I / d V$ vs. $V$ lineshapes at $R=0$.

be compared with our calculation qualitatively. We plot our calculated $|q|$, Re $q$, and the experimentally fitted $q$ in Fig. 3. The inset is a direct comparison of between our calculated and the experimental STM lineshapes at $R=0$, showing good agreement. One can see likewise that the $q$ vs. $R$ plot shows good agreement between our calculation and the experiments for $R<6 \AA$ and the discrepancy for $R>6 \AA$ is a consequence of the node of $a(R)$ around $R=7 \AA$.

The effect of the potential scattering from the Co atom is also studied by calculating the $d$-level broadening $\Delta$ and the STM lineshape prefactor $a(R)$ without potential scattering, i.e., $U_{\mathbf{k k}^{\prime}}=0$ in (3). When potential scattering is neglected, the $d$-level broadening $\Delta$ reduces by $8 \%$, and its surface-state contribution slightly increases but is still small $\left(\Delta_{\text {surf }} / \Delta \sim 0.025\right)$. We also found that without potential scattering the surface states dominate the contribution to the STM lineshape prefactor $a(R)$. This is a drastic change from the potential scattering case where bulk states dominate. This change can be understood as follows: bulk states dominate the local density of states (LDOS) of the conduction electrons at the adatom site with and without potential scattering, which accounts for the $d$-level broadening. As LDOS of the bulk states decays away from the surface faster than the surface states, in the case of no potential scattering the product of $t_{a k}$ (bulk dominated) and $t_{p k}$ (surface dominated) in (7) turns out to be dominated by the surface states. When potential scattering is included, the bare-substrate bulk and surface states strongly mix with each other. As a result, LDOS of the bulk and surface states in the presence of potential scattering decay from the surface approximately in the same rate, and $t_{a k} t_{p k}$ becomes dominated by the bulk states.

In summary, we have calculated the hybridization energies from the LDA wavefunctions of the $\mathrm{Cu}$ (111) surface and $\mathrm{Co}$ atom. The potential scattering of $\mathrm{Cu}$ conduction states from the Co adatom is included in determining the substrate-adatom hybridization energy. Our calculated $d$-level broadening from the above hybridization energy is in excellent agreement with the value determined from the STM-measured Kondo temperature. Our analysis of the contribution of the substrate-adatom hybridization energy from surface and bulk states shows that the bulk states dominate the Kondo temperature. We also calculated the tunneling conductance of an STM tip for the $\mathrm{Cu}(111)$ surface in the presence of a Co adatom. Our calculated conductance has quantitative agreement with the experiments at short parallel tip-adatom distance without any adjustable parameters. However, discrepancy appears as the parallel distance increases indicating that a new approach is required for this problem.

Acknowledgments We would like to thank O. Gunnarsson, A. Heinrich, L. Limot, V. Madhavan, J. Merino, N. Sandler, and A. Zawadowski for illuminating discussions. We would like to acknowledge support under DARPA contract no. DAAD19-01-C-0060. A.H.C.N. was partially supported through NSF grant DMR-0343790.

[1] V. Madhavan et al., Science 280, 567 (1998).

[2] H. C. Manoharan, C. P. Lutz, and D. M. Eigler, Nature (London) 403, 512 (2000).

[3] G. A. Fiete, and E. J. Heller, Rev. Mod. Phys. 75, 933 (2003).

[4] O. Agam, and A. Schiller, Phys. Rev. Lett. 86, 484 (2001).

[5] D. Porras, J. Fernández-Rossier, and C. Tejedor, Phys. Rev. B 63, 155406 (2001).

[6] N. Knorr et al., Phys. Rev. Lett. 88, 096804 (2002).

[7] L. Limot, and R. Berndt, cond-mat/0312434

[8] O. Újsághy et al., Phys. Rev. Lett. 85, 2557 (2000).

[9] M. Plihal, and J. W. Gadzuk, Phys. Rev. B 63, 085404 (2001).

[10] P. W. Anderson, Phys. Rev. 124, 41 (1961).

[11] Chiung-Yuan Lin, A. H. Castro Neto, and B. A. Jones, Phys. Rev. B 71, 035417 (2005).

[12] J. Merino, and O. Gunnarsson, Phys. Rev. Lett. 93, 156601 (2004).

[13] P. Blaha et al., WIEN2k (Karlheinz Schwarz, Techn. Universität Wien, Austria) (1999), ISBN 3-9501031-1-2.

[14] J. P. Perdew, K. Burke, and M. Ernzerhof, Phys. Rev. Lett. 77, 3865 (1996).

[15] S. D. Kevan, Phys. Rev. Lett. 50, 526 (1983); O. Jeandupeux et al., Phys. Rev. B 59, 15926 (1999).

[16] J. P. Desclaux, Comp. Phys. Commun. 9, 31(1975).

[17] P. Nordlander, and J. C. Tully, Phys. Rev. B 42, 5564 (1990).

[18] D. M. Newns, and N. Read, Adv. Phys. 36, 799 (1987).

[19] N. V. Smith, Phys. Rev. B 32, 3549 (1985).

[20] Private discussion with P. Blaha, Vienna University of Technology, Institute of Materials Chemistry.

[21] See, N. Andrei, K. Furuya, and J. Lowenstein, Rev. Mod. Phys. 55, 331 (1983), and references therein. 